\title{
Effect of various anticoagulants on the bioanalysis of drugs in rat blood: implication for pharmacokinetic studies of anticancer drugs
}

\author{
Preeti Kulkarni ${ }^{1}$, Ashwin Karanam², Murari Gurjar², Sagar Dhoble², Arvind B. Naik², Bhaskar H. Vidhun ${ }^{1}$ \\ and Vikram Gota ${ }^{2 *}$
}

\begin{abstract}
Background: Pharmacokinetic studies are vital in development and optimization of drugs. While blood samples can be collected either in EDTA, heparin or citrate containing tubes for the estimation of drug levels in plasma, EDTA tubes are more commonly used. The purpose of this study was to evaluate the effects of anticoagulants on bioanalysis of drugs. Six drugs used extensively in cancer therapy were selected. Albino wistar rats ( $N=6$ per drug) were dosed with one of the following drugs intraperitoneally — pemetrexed (50 mg/kg), imatinib (50 mg/kg), erlotinib (25 mg/ $\mathrm{kg})$, meropenem (60 mg/kg), 6-mercaptopurine $(20 \mathrm{mg} / \mathrm{kg}$ ) and voriconazole $(6 \mathrm{mg} / \mathrm{kg})$. Blood samples were collected $2 \mathrm{~h}$ after dosing ( $1 \mathrm{~h}$ in 6 -mercaptopurine group due to short half-life) by terminal bleeding from the retro-orbital plexus. Blood was collected in each of Disodium ETDA, heparin, trisodium citrate (TSC) and no anticoagulant (plain) tubes. Drug levels in these samples were determined by validated HPLC assays. ANOVA with Tukey's post hoc test was performed to identify statistically significant differences in drug concentrations in anticoagulant tubes. $p<0.05$ was considered statistically significant.
\end{abstract}

Results: Significant differences in concentration between anticoagulant tubes was observed in case of erlotinib $(p=0.013)$ and meropenem $(p=0.00)$, while borderline statistical significance for pemetrexed $(p=0.076)$. TSC tubes overestimated erlotinib levels, heparin tubes underestimated meropenem concentrations and EDTA tubes overestimated pemetrexed concentrations.

Conclusions: Careful selection of anti-coagulant is necessary for accurate characterization of pharmacokinetics of drugs. Routine use of EDTA tubes may lead to erroneous interpretation of pharmacokinetic data.

Keywords: Anticancer drugs, Bioanalysis, Plasma, Anticoagulant, Pharmacokinetics, Therapeutic drug monitoring

\section{Background}

Appropriate selection, sampling and storage of biological samples meant for estimation of drug levels is important, yet sometimes overlooked in day-to-day practice. Blood is one of the most important specimen of clinical interest as it provides unique advantages over other matrices in terms of wide variety of measurements possible, the vast amount of published data for both ante-mortem

\footnotetext{
*Correspondence: vgota76@gmail.com

${ }^{2}$ Department of Clinical Pharmacology, Advanced Centre for Treatment, Research and Education in Cancer (ACTREC), Tata Memorial Centre, Navi Mumbai, Maharashtra 410210, India

Full list of author information is available at the end of the article
}

and postmortem drug level analysis, and the interpretive value of the matrix from a pharmacological standpoint. Blood samples can be collected in tubes having a wide range of options for preservatives, anticoagulants, and other additives and subsequently stored at room temperature, refrigerated, or frozen. The purpose obviously is to maintain the sample and the drug or its metabolites of interest in a state that will not degrade from the time of collection to the time of analysis. Needless to say drug testing labs have to develop methodologies for sample analysis that allows accurate estimation and interpretation of the data. For instance, interaction between drugs and the type of collection tube used is not uncommon 
and should be accounted for during analytical method development (Boeynaems et al. 2004; Smets et al. 2004; Wang et al. 2006).

Accurate determination of drug levels in human blood samples in clinical trials and routine patient care is extremely important. For instance, therapeutic drug monitoring (TDM) guides further dose selection of a drug based on levels achieved at previous doses. If doses are modified based on incorrectly reported drug levels, it could lead to sub-therapeutic levels or potential toxicity. For anticancer drugs and some anti-microbials with narrow therapeutic window and high toxicity, appropriate determination of analyte is vital for TDM (Chantry et al. 2014; Marquet and Rousseau 2008; Paci et al. 2014).

Ethylenediaminetertraaceticacid (EDTA), heparin, and trisodiumcitrate (TSC) are the commonly used anticoagulants. However, the effect of anticoagulants on the measurement of blood drug levels is not extensively reported in literature. We therefore conducted an experiment to investigate the effect of three commonly used anticoagulants, EDTA, heparin and citric acid, on plasma drug concentration measurements of six drugs commonly used drugs in cancer care.

\section{Methods}

\section{Animals}

Albino wistar rats (weight range $250 \pm 20 \mathrm{gm}$ ) of either sex were used for the study. The experimental room was maintained under standard conditions of temperature $\left(25 \pm 2{ }^{\circ} \mathrm{C}\right)$ and relative humidity $(55 \pm 10 \%)$. Animals were subjected to $12: 12 \mathrm{~h}$ light dark cycle. Animals were housed in standard polypropylene cages with wire mesh top and husk as bedding and allowed to acclimatize for one week before the start of the study. During this period, animals were fed with commercially available rodent food pellets and water ad libitum. The experimental protocol was approved by the Institutional Animal Ethics Committee (IAEC) of Gahlot Institute of Pharmacy and the experiments were carried out in accordance with the current guidelines for the care of laboratory animals.

\section{Experimental protocol}

Six drugs commonly used in oncology practice i.e., 6-mercaptopurine (6-MP), meropenem, erlotinib, imatinib, voriconazole and pemetrexed were selected. Wistar rats $(\mathrm{N}=36)$ were divided into six groups and each group was administered one of the listed drugs intraperitoneally at doses of $50 \mathrm{mg} / \mathrm{kg}$ for pemetrexed, $50 \mathrm{mg} / \mathrm{kg}$ for Imatinib, $25 \mathrm{mg} / \mathrm{kg}$ for erlotinib, $60 \mathrm{mg} / \mathrm{kg}$ for meropenem, $20 \mathrm{mg} / \mathrm{kg}$ of $6-\mathrm{MP}$ and $6 \mathrm{mg} / \mathrm{kg}$ for Voriconazole. Blood was withdrawn from the retro orbital plexus after $2 \mathrm{~h}$. In the case of 6-MP blood sample was collected $1 \mathrm{~h}$ after dosing owing to its short half-life. The dose and time point of collection was chosen based on literature evidence and reported half-life of these drugs in rats such that the drug concentration would fall within the linearity range of our HPLC assays (Pestieau et al. 2000; Wang et al. 2015; Hoshino-Yoshino et al. 2011; Harrison et al. 1989; Tterlikkis et al. 1977; Roffey et al. 2003). Blood sample from each animal was collected in three tubes containing different anticoagulants i.e. heparin, EDTA, TSC, and a fourth collection tube that did not contain any anticoagulant (plain tube). After collection, each collection tube was centrifuged at 3000 RPM at room temperature to separate plasma and was further analyzed for drug concentrations by HPLC.

\section{Chemicals}

Acetonitrile (ACN), Methanol were procured from Sisco Research Laboratoty Pvt. Ltd, India. Dithiothreitol (DTT) and Perchloric Acid were obtained from Sigma Aldrich, USA. Dipotassium phosphate $\left(\mathrm{K}_{2} \mathrm{HPO}_{4}\right)$, Formic acid and Orthophosphoric acid were procured from S D FineChem Limited, India. Voriconazole was a kind gift of Pfizer, India. Erlotinib and Imatinib were kind gifts from Natco Pharma Limited, India. Pemetrexed was a kind gift of Dr. Reddy's Laboratories. Meropenem and 6-Mercaptopurine were procured from Sigma Aldrich, USA.

\section{Sample preparation}

The methods were developed in-house and validated according to the guidelines for validation of bioanalytical methods of FDA (Food and Drug Administration 2001). Validation was carried out for assay linearity, accuracy, precision and lower limit of quantitation (LLOQ). The coefficient of variation relative to reanalysis of the same sample was under $15 \%$ for all quality control samples of low, mid and high concentrations in the linearity range for all drugs. The following procedures were used for extracting drugs from the plasma:

\section{Imatinib}

$1000 \mu \mathrm{L}$ of ACN was added to $100 \mu \mathrm{L}$ of plasma. This was vortexed for $5 \mathrm{~min}$ and then centrifuged at 15,000 RPM for $10 \mathrm{~min}$ at room temperature. $900 \mu \mathrm{L}$ of the resulting supernatant was subsequently dried using LV Speedovap ${ }^{\circledR}$ (Takahe Analytical Instruments) for $35 \mathrm{~min}$ at $40{ }^{\circ} \mathrm{C}$. After complete drying, $100 \mu \mathrm{L}$ of premix (ACN $80 \%$, Water $20 \%$ ) was added, vortexed for $5 \mathrm{~min}$, and centrifuged at 15,000 RPM for $10 \mathrm{~min} .90 \mu \mathrm{L}$ of supernatant was transferred into autosampler vials and $30 \mu \mathrm{L}$ was injected into the HPLC.

\section{Erlotinib}

$1000 \mu \mathrm{L}$ of $\mathrm{ACN}$ was added to $100 \mu \mathrm{L}$ of plasma. This was vortexed for $5 \mathrm{~min}$ and then centrifuged at 15,000 RPM 
for $10 \mathrm{~min}$ at room temperature. $900 \mu \mathrm{L}$ of the resulting supernatant was subsequently dried using Speedovap ${ }^{\circledR}$ for $35 \mathrm{~min}$ at $40{ }^{\circ} \mathrm{C}$. After complete drying, $100 \mu \mathrm{L}$ of premix (ACN 75\%, Water 25\%) was added, vortexed for $5 \mathrm{~min}$, and centrifuged at 15,000 RPM for $10 \mathrm{~min} .90 \mu \mathrm{L}$ of supernatant was transferred into autosampler vials and $30 \mu \mathrm{L}$ was injected into the HPLC.

\section{6-Mercaptopurine}

$100 \mu \mathrm{L}$ of plasma was mixed with $50 \mu \mathrm{L}$ of DTT $(75 \mathrm{mg} /$ $\mathrm{mL}$ in water), $25 \mu \mathrm{L}$ of water and $25 \mu \mathrm{L}$ of perchloric acid. Vortexed for $5 \mathrm{~min}$ and subsequently centrifuged at $15,000 \mathrm{RPM}$ for $10 \mathrm{~min}$ at room temperature. $150 \mu \mathrm{L}$ of supernatant was kept in water bath $\left(100{ }^{\circ} \mathrm{C}\right)$ for $45 \mathrm{~min}$, after which it was cooled to room temperature and transferred into autosampler vials and $30 \mu \mathrm{L}$ was injected into the HPLC.

\section{Voriconazole}

Briefly, $800 \mu \mathrm{L}$ of ACN was added to $100 \mu \mathrm{L}$ of plasma. Vortexed for $5 \mathrm{~min}$ and subsequently centrifuged at $15,000 \mathrm{RPM}$ for $10 \mathrm{~min}$ at room temperature. $100 \mu \mathrm{L}$ of supernatant was mixed with $100 \mu \mathrm{L}$ Buffer solution (10 mM Ammonium Acetate, $5 \mathrm{pH}$ ), followed by vortexing for $2 \mathrm{~min}$ and then centrifugation at 15,000 RPM for $10 \mathrm{~min} .100 \mu \mathrm{L}$ of supernatant was transferred into autosampler vials and $50 \mu \mathrm{L}$ was injected into the HPLC.

\section{Meropenem}

Solid Phase extraction was done using SPE cartridges. SPE cartridges were washed with $1 \mathrm{~mL}$ methanol followed by equilibration with $1 \mathrm{~mL}$ equilibration buffer $(100 \mathrm{mM}$ $\mathrm{K}_{2} \mathrm{HPO}_{4} \mathrm{pH}$ 6.2). Plasma $(100 \mu \mathrm{L})$ were then loaded into the cartridges. Secondary washing was performed with $500 \mu \mathrm{L}$ of an equilibration buffer ( $\mathrm{pH}$ 6.2). The samples were then eluted with $500 \mu \mathrm{L}$ of $100 \%$ ACN followed by drying the eluent by Speedovap ${ }^{\circledR}\left(40-45{ }^{\circ} \mathrm{C}\right)$. After drying, sample was reconstituted with premix $(100 \mu \mathrm{L})$, vortexed for $2 \mathrm{~min}$ and centrifuged at 12,000 RPM. [Premix-A:B (5:95) where, $\mathrm{A}=\mathrm{ACN}+$ Methanol (50:50), $\mathrm{B}=\mathrm{KH}_{2} \mathrm{PO}_{4}$ Buffer $\mathrm{pH}$ 6.2]. $90 \mu \mathrm{L}$ of the sample were loaded into HPLC vials and $30 \mu \mathrm{L}$ was injected into the column.

\section{Pemetrexed}

Solid phase extraction was done using SPE cartridge. SPE cartridges were conditioned with $0.5 \mathrm{~mL}$ methanol followed by equilibration with $0.5 \mathrm{~mL}$ of $100 \mathrm{mM} \mathrm{K}_{2} \mathrm{HPO}_{4}$ ( $\mathrm{pH}$ 6.2). Samples were then loaded in the cartridges. Secondary washing was done with $0.5 \mathrm{~mL}$ of $100 \mathrm{mM}$ $\mathrm{K}_{2} \mathrm{HPO}_{4}$, pH 6.2 followed by $100 \%$ ACN $(250 \mu \mathrm{L})$. Plasma $(100 \mu \mathrm{L})$ was subsequently eluted with $0.5 \mathrm{~mL}$ of Formic acid: methanol (5:95). These were dried by Speedovap ${ }^{\circledR}$ $\left(40{ }^{\circ} \mathrm{C}\right.$ ) for $40 \mathrm{~min}$. After drying, samples were reconstituted with $100 \mu \mathrm{L}$ of $100 \mathrm{mM} \mathrm{K}_{2} \mathrm{HPO}_{4}(\mathrm{pH}$ 6.2), vortexed for $5 \mathrm{~min}$ and centrifuged for $10 \mathrm{~min}$ at 15,000 RPM. $90 \mu \mathrm{L}$ of this supernatant was loaded into HPLC vials and $30 \mu \mathrm{L}$ was injected into the column.

\section{HPLC conditions}

HPLC analysis was carried out under the conditions mentioned in Table 1. HPLC model: Dionex UHPLC Ultimate 3000 with diode array detector Dionex Ultimate 3000 RS was used. Kinetex ${ }^{\circledR}$ (Phenomenex, USA) columns were used in the analysis.

\section{Statistical analysis}

All statistical tests were performed using SPSS 20 (IBM). The samples collected in plain tubes (without any anticoagulant) were considered as control group and the concentrations observed in anticoagulant tubes were normalized against this value. We define 'plain normalization' as ratio of concentration of the drugs in each of the anticoagulant tubes with corresponding concentrations in plain tubes of the same animal. This normalization was carried out in order to eliminate interanimal variability in pharmacokinetics, thus making it possible to attribute differences in plain normalized values between anticoagulant groups to the anticoagulant effect. ANOVA, with Tukey's post hoc test was used to compare the plain normalized values between the anticoagulant groups. A p value of less than 0.05 was considered statistically significant.

\section{Results and discussion}

Validation parameters including linearity, accuracy, precision and LLOQ for each analyte is shown in Table 2. The concentration of drugs determined using different anticoagulants is presented in Fig. 1 (The raw data showing concentration of each drug observed in each animal for different anticoagulants may be found in Additional file 1). As seen from the figure, we observed significant inter-animal variability within the same anticoagulant group. The plain normalized ratios are presented in Fig. 2, depicting a tighter set of observations by eliminating interanimal variability, and thereby bringing anticoagulant effect to the fore as in the case of erlotinib and meropenem. ANOVA test revealed statistically significant differences in concentration in case of erlotinib $(\mathrm{p}=0.013)$ and meropenem $(\mathrm{p}<0.01)$ while borderline statistical significance for pemetrexed $(p=0.076)$ between various anticoagulants as seen in Fig. 3. Between group comparison using Tukey's post hock test showed that heparin tubes significantly underestimated meropenem concentrations when compared to EDTA (Tukey, $\mathrm{p}=0.001)$ and TSC tubes (Tukey, $\mathrm{p}=0.003$ ) (Fig. 3a). 


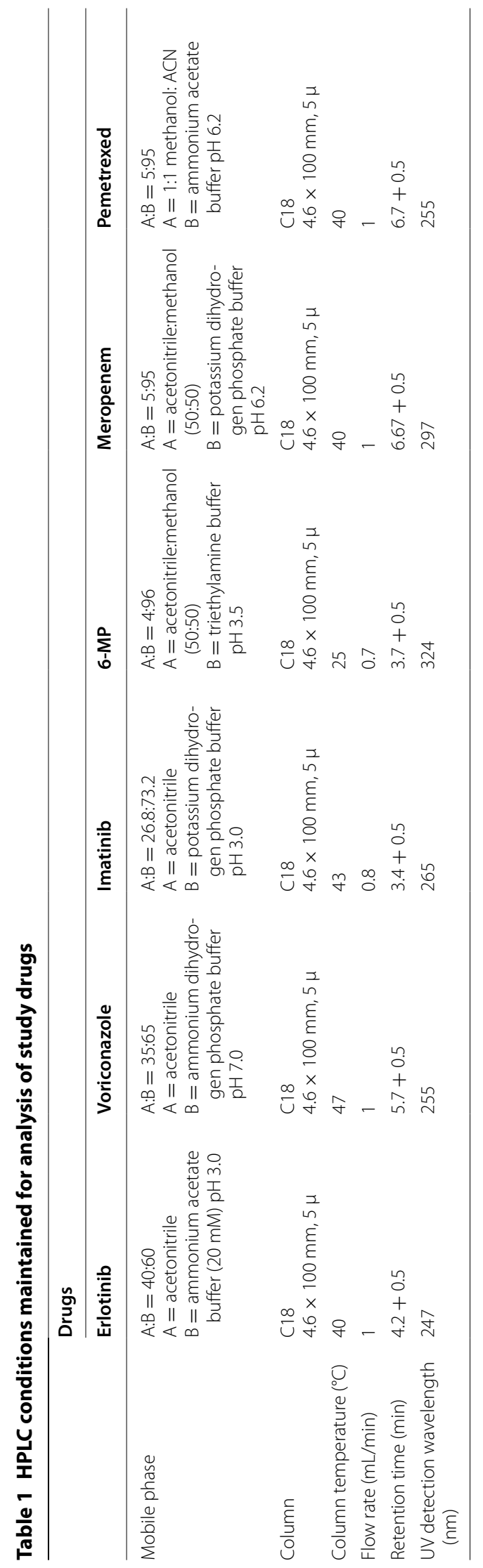


Table 2 Validation parameters of linearity, accuracy, precision and LLOQ for each analyte

\begin{tabular}{lllll}
\hline Analyte & Linearity range $(\boldsymbol{\mu g} / \mathbf{m L})$ & Accuracy* & Precision* $(\mathbf{C V} \%)$ & LLOQ $(\boldsymbol{\mu g} / \mathbf{m L})$ \\
\hline Erlotinib & $0.1-10$ & $95.6-98.7$ & $3.7-10.5$ & 0.1 \\
Pemetrexed & $0.1-10$ & $96.2-101.1$ & $2.9-12.1$ & 0.1 \\
Voriconazole & $0.25-8$ & $100.4-102.3$ & $6.0-9.7$ & 0.25 \\
Imatinib & $0.1-10$ & $103.3-101.7$ & $3.4-5.7$ & 0.1 \\
6MP & $0.1-10$ & $102.4-107.4$ & $4.6-6.1$ & 0.1 \\
Meropenem & $0.1-10$ & $89.5-97.2$ & $4.3-4.5$ & 0.1 \\
\hline
\end{tabular}

CV coefficient of variation, LLOQ lower limit of quantitation

* Range of accuracy and precision values shown for low, medium and high QC samples

The significance of this observation lies in the fact that meropenem is a time-dependent antibiotic which exerts antibacterial activity more efficiently if the plasma concentration is more than the Minimum Inhibitory Concentration (MIC) of the targeted strain of bacteria for at least $40 \%$ of the dosing interval (fT > MIC > 40\%) (Drusano 2003). Under such circumstances, using heparin tubes for blood collection may grossly underestimate the fraction of time above MIC (fT > MIC) resulting in unnecessary dose modifications. In case of erlotinib, TSC tubes significantly overestimated the concentration of the drug in comparison to heparin (Tukey, $\mathrm{p}=0.019$ ) and EDTA tubes (Tukey, $\mathrm{p}=0.028$ ) (Fig. 3b). In case of pemetrexed, concentrations in the EDTA tubes tended to be higher than heparin tubes but the difference was not statistically significant (Tukey, $\mathrm{p}=0.069$ ) (Fig. 3c). Anticoagulant effect was not observed in the case of voriconazole, imatinib and 6-MP.

Commonly used anticoagulants for blood collection include EDTA, heparin and citrate, EDTA being used most frequently. However, EDTA is a known chelator and hence not used in estimation of metal ions. Cisplatin for example, is collected in heparin coated tubes. Identification of such interactions is key to minimizing influence of any external factors on the analyte concentrations. Barring a few examples, mechanisms by which anticoagulants affect the measurement of drug concentrations is poorly understood. A study similar to ours was performed by Chen et al. (2008) with tigecyclin and ciprofloxacin in rats, using EDTA and heparin collection
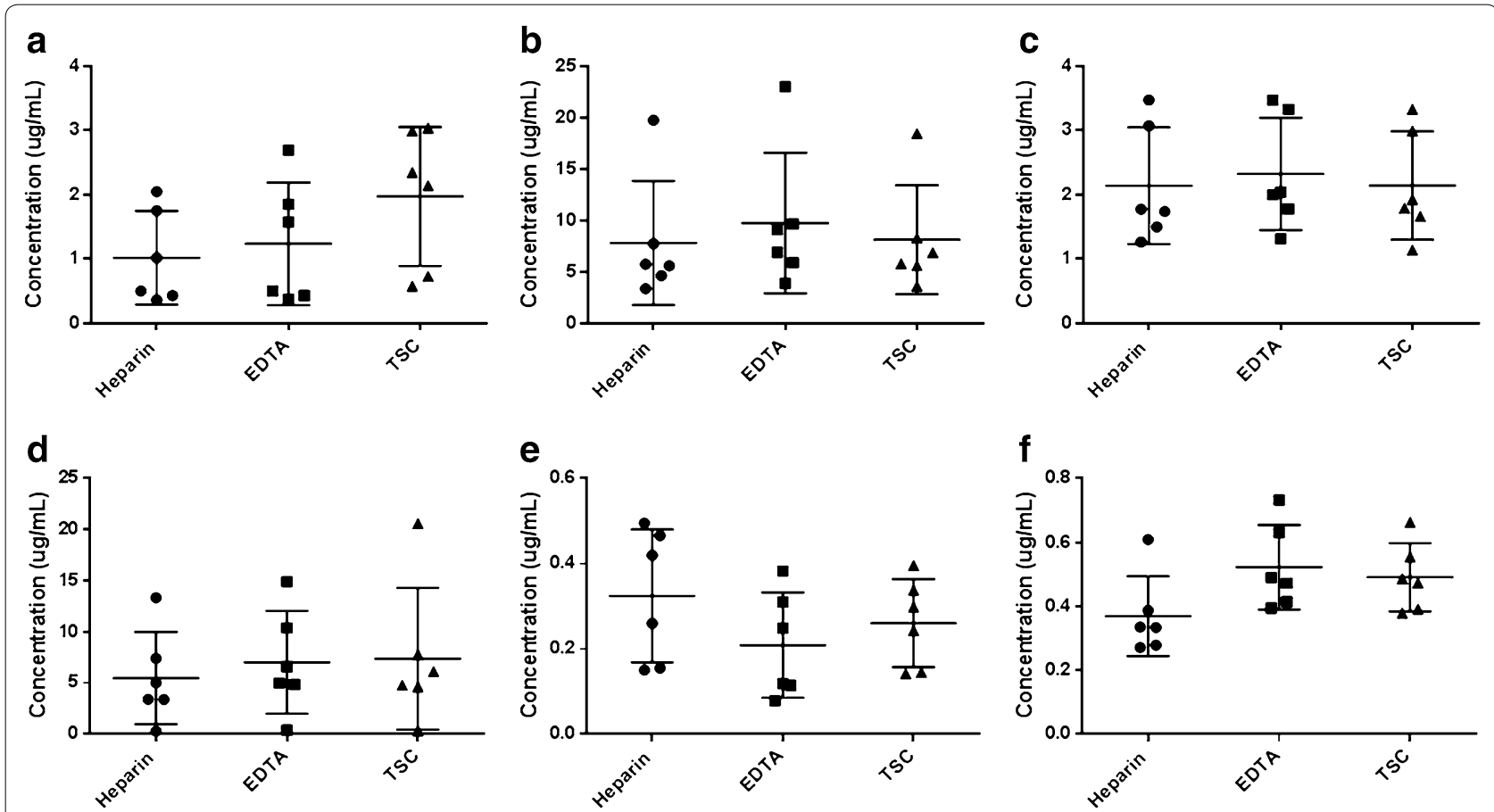

Fig. 1 Individual plasma concentrations of $\mathbf{a}$ erlotinib, $\mathbf{b}$ pemetrexed, $\mathbf{c}$ voriconazole, $\mathbf{d}$ imatinib, e 6-mercaptopurine and $\mathbf{f}$ meropenem when collected in heparin coated, EDTA coated and TSC coated tubes 

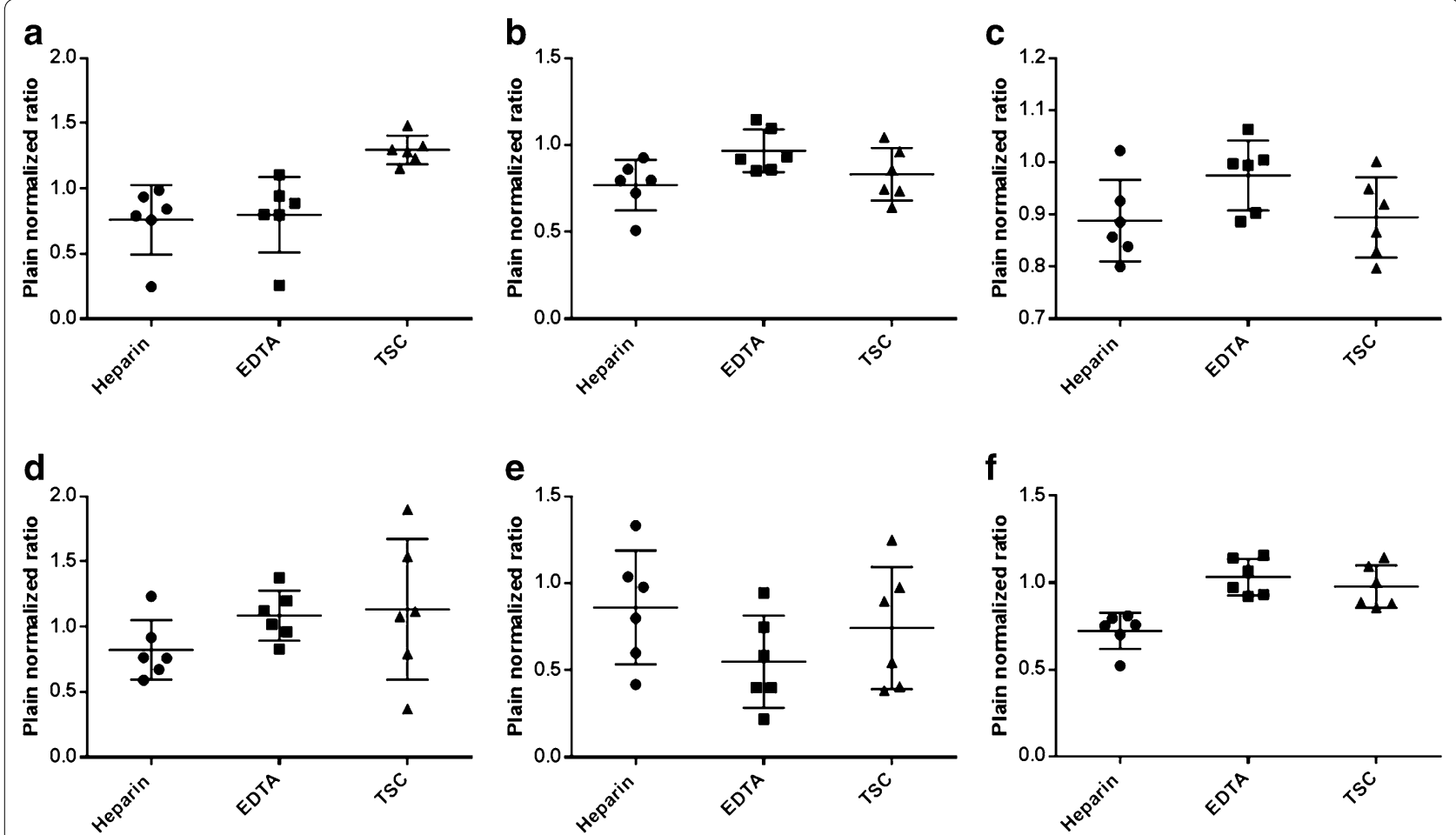

Fig. 2 Individual plain normalized ratios of $\mathbf{a}$ erlotinib, b pemetrexed, $\mathbf{c}$ voriconazole, $\mathbf{d}$ imatinib, e 6-mercaptopurine and $\mathbf{f}$ meropenem when collected in heparin coated, EDTA coated and TSC coated tubes
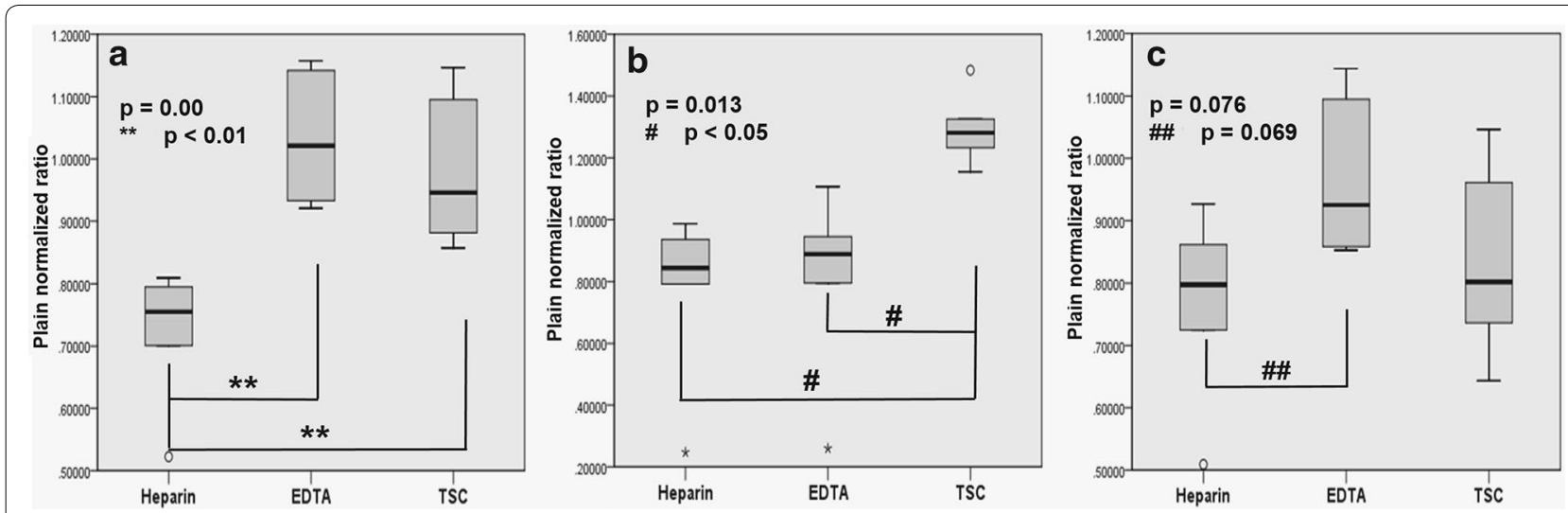

Fig. 3 Box and whisker plots of plain normalized ratios of a pemetrexed, $\mathbf{b}$ meropenem, $\mathbf{c}$ erlotinib depicting statistical significance at indicated $\mathrm{p}$ values

tubes. They concluded that EDTA might compete with tigecyclin and ciprofloxacin for chelating metal ions, thereby affecting drug partitioning in the plasma compartment leading to inaccurate estimation of drug levels.

Accurate detection of analyte concentrations is one of the most important challenges faced in clinical setting. The FDA Guidance to Industry for bioanalytical method validation necessitates evaluation of matrix effect (including anticoagulants) before the method can be adopted for bioanalysis of drugs, however there are no guidelines pertaining to how these effects should be evaluated Food and Drug Administration (2001). This is often overlooked in non-regulatory studies which constitute the major segment of published literature, thereby perpetuating errors in the estimation of pharmacokinetic parameters of drugs. Our findings provide compelling argument in favor of identifying the compatibility of anticoagulants with the analytes. To the best of 
our knowledge, this is the first study which has systematically evaluated anticoagulant effects on the bioanalysis of drugs used in cancer care. Since some of these drugs are good candidates for pharmacokinetics guided dose optimization or TDM (Moriyama et al. 2015; De Keukeleire et al. 2016), and there is evidence emerging in case of some others (Herviou et al. 2016), the findings of this study clearly underscore the importance of choosing the right anticoagulant for pharmacokinetic studies and bioanalysis of TDM samples. The findings are also relevant to other fields including forensic medicine where plasma drug levels are often analyzed.

The importance of type of anticoagulant coated collection tube does not only apply for drugs and metabolite concentrations, but has to be extended to other biological analytes as well. The influence of various anticoagulants in biochemical analytes have been substantiated by several studies. Heparin has been identified to interfere with estimation of thyroid hormones and albumin levels (Bowen and Remaley 2014). Chuang et al. (1998) described the influence of anticoagulants in amino acid analysis. They identified that EDTA reacts with ninhydrin reagent used in amino acid analysis and produces a ninhydrin positive contaminant, which interferes with the analysis. Similarly, they suggested that heparin coated tubes may interfere with estimation of sulfur containing amino acids due to presence of a preservative, sodium metabisulfite. Suttnar et al. (2001) demonstrated the underestimation of malonaldehyde levels (a marker of lipid peroxidation), when collected in EDTA tubes. Lykkesfeldt (2012) noted that use of EDTA tubes prevented ex vivo oxidation of oxidative stress biomarkers ascorbate and dehydroascorbic acid when compared to 5 other anticoagulants. Mohri and Rezapoor (2009) also demonstrated the difference in estimating routine biochemical parameters using EDTA, heparin and citrate tubes. The chelating nature of EDTA and its influence on samples collected for blood clotting assay and serum electrolytes was shown by Lima-Oliveria et al. (2014, 2015). Cross-contamination of heparinized or citrated blood with EDTA was shown to adversely affect the blood clotting assay and also the electrolyte levels, grossly underestimating them. A study by Wiese et al. (1997) recommended the use of EDTA or heparin tubes over citrate tubes as they led to lower lactate concentration measurement in critically ill.

The spectrum of influence of anticoagulants on analytes is not only limited to interference with their concentrations, but also in analyte stability. More often than not, blood samples are not processed immediately. Rather, they are refrigerated and are then analyzed at a later point of time. This was elucidated by Lam et al. (2004) for DNA analysis, wherein they suggested use of EDTA as anticoagulant of choice for delayed blood processing.
Some limitations of our study include a small sample size leading to lack of statistical power. However, the study still manages to establish the proof of concept in spite of this limitation. Secondly, we did not use internal standards (IS) in our HPLC assays since we did not want to confound our observations by introducing another factor (the IS) which may, on its own, be influenced by the anticoagulant effect. Instead, we relied on robust validation of the assays as per FDA bioanalytical validation guidelines.

\section{Conclusions}

To conclude, the choice of anticoagulant influenced the estimation of plasma concentration for three out of 6 drugs studied. Influence of anticoagulants on the estimation of drug levels should be a guiding factor in choosing an appropriate anticoagulant for accurate characterization of pharmacokinetics of drugs. EDTA as the anticoagulant of choice is questionable and may lead to erroneous interpretation of pharmacokinetic data in some cases.

\section{Additional file}

Additional file 1. Raw data showing concentration observed for each drug in each animal following collection in EDTA, heparin, TSC and plain tubes.

\begin{abstract}
Abbreviations
TSC: trisodium citrate; EDTA: ethylenediaminetertraaceticacid; IAEC: Institutional Animal Ethics Committee; 6-MP: 6-mercaptopurine; ACN: acetonitrile; DTT: dithiothreitol; LLOQ: lower limit of quantitation; MIC: minimum inhibitory concentration; fT > MIC: fraction of time above MIC; TDM: therapeutic drug monitoring.
\end{abstract}

\section{Authors' contributions}

PK conceived of the study, obtained the research grant and animal ethics approval and participated in design and coordination of the study. AK performed the statistical analysis and drafted the manuscript. MG carried out bioanalysis of samples on HPLC. SD carried out the animal studies and drafted the manuscript. ABN carried out the animal studies. BHV participated in design and coordination of the study and helped to draft the manuscript. VG conceived of the study, participated in its design and helped to draft the manuscript. All authors read and approved the final manuscript.

\section{Author details}

${ }^{1}$ Gahlot Institute of Pharmacy, Plot No 59, Sector 14, Koparkhairne, Navi Mumbai, Maharashtra, India. ${ }^{2}$ Department of Clinical Pharmacology, Advanced Centre for Treatment, Research and Education in Cancer (ACTREC), Tata Memorial Centre, Navi Mumbai, Maharashtra 410210, India.

\section{Competing interests}

The authors declare that they have no competing interests.

\section{Ethics approval and consent to participate}

The experimental protocol was approved by the Institutional Animal Ethics Committee (IAEC) of Gahlot Institute of Pharmacy, Mumbai, India.

\section{Funding}

The study was supported by a Grant No. APD/237/369 of 2013 from University of Mumbai. The funding body had no role in the design of the study and collection, analysis, and interpretation of data and in writing the manuscript. 


\section{Statement on the welfare of animals}

All applicable international, national, and institutional guidelines for the care and use of animals were followed.

Received: 11 June 2016 Accepted: 30 November 2016

Published online: 20 December 2016

\section{References}

Boeynaems JM, De Leener A, Dessars B, Villa-Lobos HR, Aubry JC, Cotton F et al (2004) Evaluation of a new generation of plastic evacuated blood-collection tubes in clinical chemistry, therapeutic drug monitoring, hormone and trace metal analysis. Clin Chem Lab Med CCLM/FESCC 42(1):67-71. doi:10.1515/CCLM.2004.013

Bowen RA, Remaley AT (2014) Interferences from blood collection tube components on clinical chemistry assays. Biochem Med (Zagreb) 24(1):31-44. doi:10.11613/BM.2014.006

Chantry AS, Quaranta S, Ciccolini J, Lacarelle B (2014) Clinical application, limits and perspectives of pharmacogenetic and pharmacokinetic analysis of anticancer drugs. Ann Biol Clin 72(5):527-542. doi:10.1684/abc.2014.0993

Chen Q, Tung EC, Ciccotto SL, Strauss JR, Ortiga R, Ramsay KA et al (2008) Effect of the anticoagulant ethylenediamine tetra-acetic acid (EDTA) on the estimation of pharmacokinetic parameters: a case study with tigecycline and ciprofloxacin. Xenobiotica 38(1):76-86. doi:10.1080/00498250701678955

Chuang CK, Lin SP, Lin YT, Huang FY (1998) Effects of anticoagulants in amino acid analysis: comparisons of heparin, EDTA, and sodium citrate in vacutainer tubes for plasma preparation. Clin Chem 44(5):1052-1056

De Keukeleire S, Borrey D, Decaluwe W, Reynders M (2016) Therapeutic drug monitoring of meropenem in neonate with necrotizing enterocolitis: a challenge. Case Rep Infect Dis 2016:6207487

Drusano GL (2003) Prevention of resistance: a goal for dose selection for antimicrobial agents. Clin Infect Dis 36(Suppl 1):S42-S50

Food and Drug Administration (2001) Guidance for industry: bioanalytical method validation. In: Research CfDEa, editor

Harrison MP, Moss SR, Featherstone A, Fowkes AG, Sanders AM et al (1989) The disposition and metabolism of meropenem in laboratory animals and man. J Antimicrob Chemother 24(Suppl A):265-277

Herviou P, Thivat E, Richard D, Roche L, Dohou J, Pouget M et al (2016) Therapeutic drug monitoring and tyrosine kinase inhibitors. Oncol Lett 12(2):1223-1232

Hoshino-Yoshino A, Kato M, Nakano K, Ishigai M, Kudo T, Ito K (2011) Bridging from preclinical to clinical studies for tyrosine kinase inhibitors based on pharmacokinetics/pharmacodynamics and toxicokinetics/toxicodynamics. Drug Metab Pharmacokinet 26(6):612-620. doi:10.2133/dmpk. DMPK-11-RG-043

Lam NY, Rainer TH, Chiu RW, Lo YM (2004) EDTA is a better anticoagulant than heparin or citrate for delayed blood processing for plasma DNA analysis. Clin Chem 50(1):256-257. doi:10.1373/clinchem.2003.026013
Lima-Oliveira G, Salvagno GL, Danese E, Brocco G, Guidi GC, Lippi G (2014) Contamination of lithium heparin blood by K2-ethylenediaminetetraacetic acid (EDTA): an experimental evaluation. Biochem Med (Zagreb) 24(3):359-367. doi:10.11613/BM.2014.038

Lima-Oliveira G, Salvagno GL, Danese E, Favaloro EJ, Guidi GC, Lippi G (2015) Sodium citrate blood contamination by K2 -ethylenediaminetetraacetic acid (EDTA): impact on routine coagulation testing. Int J Lab Hematol 37(3):403-409. doi:10.1111/ijlh.12301

Lykkesfeldt J (2012) Ascorbate and dehydroascorbic acid as biomarkers of oxidative stress: validity of clinical data depends on vacutainer system used. Nutr Res 32(1):66-69. doi:10.1016/j.nutres.2011.11.005

Marquet P, Rousseau A (2008) Pharmacokinetics and therapeutic drug monitoring of anticancer agents. Bull Cancer 95(10):903-909. doi:10.1684/ bdc.2008.0725

Mohri M, Rezapoor H (2009) Effects of heparin, citrate, and EDTA on plasma biochemistry of sheep: comparison with serum. Res Vet Sci 86(1):111114. doi:10.1016/j.rvsc.2008.05.010

Moriyama B, Kadri S, Henning SA, Danner RL, Walsh TJ, Penzak SR (2015) Therapeutic drug monitoring and genotypic screening in the clinical use of voriconazole. Curr Fungal Infect Rep 9(2):74-87

Paci A, Veal G, Bardin C, Leveque D, Widmer N, Beijnen J et al (2014) Review of therapeutic drug monitoring of anticancer drugs part 1-cytotoxics. Eur J Cancer 50(12):2010-2019. doi:10.1016/j.ejca.2014.04.014

Pestieau SR, Stuart OA, Sugarbaker PH (2000) Multi-targeted antifolate (MTA): pharmacokinetics of intraperitoneal administration in a rat model. Eur J Surg Oncol 26(7):696-700

Roffey SJ, Cole S, Comby P, Gibson D, Jezequel SG, Nedderman AN et al (2003) The disposition of voriconazole in mouse, rat, rabbit, guinea pig, dog, and human. Drug Metab Dispos 31(6):731-741

Smets EM, Dijkstra-Lagemaat JE, Blankenstein MA (2004) Influence of blood collection in plastic vs. glass evacuated serum-separator tubes on hormone and tumour marker levels. Clinical Chem Lab Med CCLM/FESCC 42(4):435-439. doi:10.1515/CCLM.2004.076

Suttnar J, Masova L, Dyr JE (2001) Influence of citrate and EDTA anticoagulants on plasma malondialdehyde concentrations estimated by highperformance liquid chromatography. J Chromatogr B Biomed Sci Appl 751(1):193-197

Tterlikkis L, Ortega E, Solomon R, Day JL (1977) Pharmacokinetics of mercaptopurine. J Pharm Sci 66(10):1454-1457

Wang S, Ho V, Roquemore-Goins A, Smith FA (2006) Effects of blood collection tubes, including pediatric devices, on 16 common immunoassays. Clin Chem 52(5):892-893. doi:10.1373/clinchem.2006.068361

Wang Z, Wang L, Xia MM, Sun W, Huang CK, Cui X et al (2015) Pharmacokinetics interaction between imatinib and genistein in rats. Biomed Res Int 2015:368976. doi:10.1155/2015/368976

Wiese J, Didwania A, Kerzner R, Chernow B (1997) Use of different anticoagulants in test tubes for analysis of blood lactate concentrations: Part 2. Implications for the proper handling of blood specimens obtained from critically ill patients. Crit Care Med 25(11):1847-1850

\section{Submit your manuscript to a SpringerOpen ${ }^{\circ}$ journal and benefit from:}

- Convenient online submission

- Rigorous peer review

- Immediate publication on acceptance

- Open access: articles freely available online

- High visibility within the field

- Retaining the copyright to your article

Submit your next manuscript at $\boldsymbol{\nabla}$ springeropen.com 